EP-170

\title{
Organ preserving pancreatic resections offers better long-term conservation of pancreatic function at the expense of significantly high perioperative major morbidity: A fair trade-off for benign or low malignant potential pancreatic neoplasms
}

\author{
Vikas GUPTA*, Manish BHANDARE, Vikram CHAUDHARI, Shailesh SHRIKHANDE
}

Department of Surgical Oncology, TATA Memorial Hospital, Mumbai, India

Introduction: Standard pancreatic resections often remove excess pancreatic parenchyma than required for tumors of low malignant potential (LMP) or benign neoplasms. We aimed to compare short and long-term outcomes following organ-ppreserving pancreatic resections (OPPAR) and standard pancreatic resections (SPR).

Methods: Data was collected from a prospectively maintained database of patients who underwent OPPAR or SPR for benign or LMP pancreatic tumors from January 2011 to January 2020 at Tata Memorial Hospital.

Results: 40 and 110 patients were identified in OPPAR and SPR groups respectively. The mean surgery duration (277 vs. 339 minutes, $p=0.006$ ) and mean blood loss (602 vs $937 \mathrm{~mL}, p=0.01$ ) were significantly lower in patients who underwent OPPAR. Although the overall morbidity $(57.5 \%$ vs $43.6 \%, p=0.13)$ was comparable, the major morbidity ( $42.5 \%$ vs $20.9 \%, p=0.008)$, post-operative pancreatic fistula (POPF) (65\% vs $33.6 \%, p=0.001)$ and clinically relevant POPF ( $42.5 \%$ vs. $19.1 \%, p=0.004)$ were significantly higher in OPPAR. After a median follow up of 29 months the post operative endocrine insufficiency ( $10 \%$ vs $15.5 \%, p=0.39$ ), exocrine insufficiency ( 20 $\%$ vs. $0 \%, p=0.002)$ and requirement of long-term pancreatic enzyme replacement $(18.2 \%$ vs. $0 \%, p=0.004)$ were higher in SPRs. Left sided SPRs had higher endocrine insufficiency $(17.1 \%$ vs. $11.2 \%, p=0.29)$ and right sided SPRs had higher exocrine insufficiency $(20 \%$ vs. $8.6 \%, p=0.04)$.

Conclusions: OPPAR should be considered for treatment of benign tumors and tumors of LMP with favorable features. Although the incidence of post-operative major morbidity and POPF is higher with OPPAR than standard resections, there appears to be long term functional benefit. 